\title{
TPL: NOVEL Efficient Threshold Power Consumption Scheme on MANET
}

\author{
Hatem S. A. Hamatta \\ Dept. of Applied Sciences \\ Al-Balqa Applied University
}

\author{
Mohammad U. Bokhari \\ Chairman, Department of \\ Computer Science \\ Aligarh Muslim University
}

\author{
Shams Tabrez Siddigui \\ Research Scholar, Department \\ of Computer Science \\ Aligarh Muslim University
}

\begin{abstract}
Wireless nodes (Laptop, PDA's and Mobiles) are assumed to have transmission power with different discrete level and different control to the limit of power of usage. The level of power available is one of the main concepts which affect all of ad hoc wireless networks. Since, all of these networks are constructed without any infrastructure, where it based on the mobility of nodes that prevents the usage of any fixed infrastructure such as power cables. Hence, the power consumption is an important factor which affects the network life-time and transmission range. For that, the power consumption rate must be distributed for every node and the overall power of transmission must be minimized for each connection request, also the congestions must be decreased. A clustering scheme is used in the adaptation of MANETs to be energy efficient. For this motivation, this paper presents a simple scheme which maintains the idea of Cluster Head election that allows mobile nodes to automatically create best clusters that use an optimal scenario of cluster formation and maintenance which conserve the power energy consumptions, and increase lifetime of ad hoc wireless network's devices. The main idea is to partition the network into set of clusters, and also find the best scale of clusters according to power level of all nodes in each cluster.
\end{abstract}

\section{General Terms}

Mobile Ad Hoc Network, Clustering Algorithms and Energy.

\section{Keywords}

Power consumption, Clustering, MANETs, Cluster Head, Ordinary Node and Network Lifetime.

\section{INTRODUCTION}

Recently, many researches and studies covers wireless Ad Hoc Networks as a non-infrastructure network with dynamic behavior to formulate the network environment. The network is automatically established and updates connectivity with other devices available in the network. Many research defined the main characteristic of Wireless Ad Hoc Networks as a network environments in which most of its users are mobiles, which provides dynamic topology changes. This indicates no stable communication infrastructure [14]. This characteristic derives many factors which affect this kind of networks, such as power limitation (batteries), mobility, etc. Wireless Ad Hoc Networks can be classified according to their application, type of component or internal design into the following categories [7]:
1. Wireless Sensor Networks.

2. Wireless Mesh Networks.

3. Mobile Ad Hoc Networks.

This paper focus on improvement of Mobile Ad Hoc Networks lifetime by decreasing the power energy consumption through the idea derived from clustering techniques. The reset of this paper is organized as follows; Section 2 reviews existing literatures of power consumption and clustering algorithms in MANETs. Section 3 presents the proposed clustering scheme. Finally, conclusion and future research work are presented in section 4 .

\section{Background and Related Work}

Mobile Ad Hoc Network can be defined as a self-configuring platform network of mobile devices connected by wireless links. This kind of network faces primary challenges in route discovery and information maintenance i.e. properly routing traffic [18] [25]. This kind of networks may operate by themselves i.e. Internet Based Mobile Ad Hoc Networks (iMANET) or may connect to larger internet i.e. Vehicular Ad Hoc Networks (VANETs). This mean that the system may operate in isolation or may have gateway to and interface with fixed network.

All Mobile Ad Hoc Networks devices share the same property i.e., all are working on batteries, which make it the most affected factor on this kind of network that specify the overall network lifetime. If any of nodes is out of battery energy, the entire network goes down.

\subsection{Energy Efficiency}

Mobile Ad Hoc Networks devices works on either replaceable or rechargeable batteries. Therefore, the batteries provide an energy quantity very limited to all nodes on scale. Also, the node will consume its energy while transmitting or receiving messages or even when it is idle. So, these reasons limit the lifetime of the device which influences the total network operations and lifetime. For this reason why, today, techniques ensuring the level of low energy consumption occupy an important research direction in this field [9] [11].

The energy efficiency continues to be a key factor in limiting the deployability of MANETs. Maximum lifetime of the networks has been exploited by deploying an energy efficient system. Hence, the total network lifetime is the time period from the instance at which the network functionality start to the time instant at which any node run out of its energy [18]. Therefore, the greatest clear challenge in designing wireless ad-hoc networks is the limited availability of the energy resources. 
Some algorithms [17] reduced the level of consumed energy by producing single path routing discovery process in MANETs by working on DSR [12] or AODV [19], while others takes several multi-path protocols for wireless ad-hoc networks such as the Ad-hoc On Demand Distance Vector Multi-path routing protocol (AODVM) [27] and Split Multipath Routing (SMR) [15] have been proposed. The Ad hoc On-demand Multi-path Distance Vector protocol (AOMDV) is an extension of AODV for discovering node-disjoint or optionally link-disjoint paths [16].

\subsection{Clustering}

The most popular methods used to provide resource management on MANETs is based on partitioning the network into small manageable groups of nodes called clusters [21]. Clustering is the most common technique which offers several advantages in MANETs, like:

- Improving routing and mobility.

- Providing virtual infrastructure for dynamic network.

- $\quad$ Stabilizing the network topology.

- Helping to perform more efficient resource allocation.

Also it used in hierarchical routing architecture to divide the nodes of a self-organized network into a number of overlapping or disjoint clusters. Thereby, Cluster-Based System classified the nodes into three types as shown in Figure 1 [23] defined as follows:

Cluster Head: is a local coordinator for its cluster, which performing inter-cluster routing, data forwarding and many other operations.

Gateway Node: is a Non-Cluster-Head node which shared between more than one clusters. Thus it can access neighboring cluster and forward information between clusters.

Normal or Ordinary Nodes: is a node that is neither a Cluster Head nor a Cluster Gateway. Also it can be called (Cluster Member).

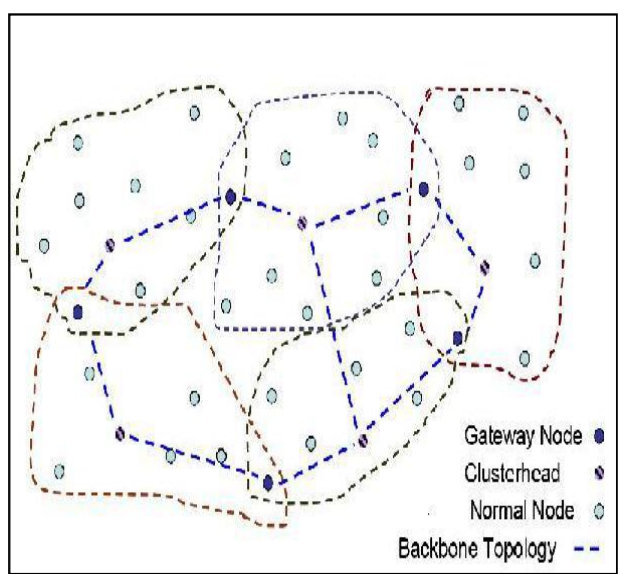

Fig 1: Clustering Network in MANETs

In any cluster one node must be elected to be a Cluster Head to maintain the membership information for the cluster. Nodes that are not Cluster Head will, henceforth, be referred to as Ordinary Nodes except that node act as a shared node between different clusters called Gateway Nodes. The Cluster Head needs to coordinate all transmission within the cluster, also it handles all traffic and delivery of the packets designated for the cluster. For this reason, the Cluster Head consumes high level of its energy and thereby exhausted more quickly than Cluster Members. Clustering deployment consists two phases:

- Cluster Formulation.

- Cluster Maintenance.

Clustering algorithms have been proposed in several techniques according the way of grouping nodes into clusters [1] [4] [6] [10] [13]. All researchers agreed that the cluster formation and maintenance is a most important term in power consumption in MANETs. For this reason, some of the most important cluster formation schemes are briefly reviewed with their features as follows:

\subsubsection{Lowest-ID Clustering}

The lowest-ID clustering is the simplest clustering scheme [8] [10]. In this scheme every node in the network distinguished by unique IDentifier (ID). All nodes broadcast their ID periodically and the node with the lowest ID decides to become a Cluster Head. The main disadvantage with this scheme is there is no limit to the number of nodes connected to the same network. And hence, the power limitation can appear without any respect to overall network lifetime since no network consideration is given in selection for the Cluster Head.

\subsubsection{DS Based Clustering}

Researcher [5] [26] proposed techniques which based on Dominating Set (DS) of nodes that acts as Cluster Heads and all non-Cluster-Head is assigned only to one Cluster Head. As previous structure, this has the same drawback of Lowest-ID because its Cluster Head Dissipates more power compared with other nodes in the cluster. This situation needs reelection process after the Cluster Head out of service or moves out of the range of the cluster.

\subsubsection{Highest Degree Clustering}

In contrast to Lowest-ID scheme, the Highest Degree Clustering takes into account the degree of a node [8]. A node with highest degree (i.e. the number of its directly connected neighbors by one hop) is selected as Cluster Head. This technique follows the same circumstances of Lowest-ID according to cluster size and performance degradation.

\subsubsection{Mobility Based and Weight Clustering}

This clustering scheme is similar to the Lowest-ID except that it keeping mobility as a metric for cluster formation. MOBIC as proposed by [2-3] uses a new mobility metric, it takes into account the aggregate of local mobility as a metric to provide the election in cluster construction process. In this metric as described in [2], every node in the network transmit "Hello" periodic message to its neighbors. Each node measure the relative mobility between its neighboring nodes. After that, each node calculates aggregate mobility by calculating the value of variance and broadcasts it to the other nodes. The node with the lowest aggregate mobility elected as Cluster Head.

This technique falling under two main drawbacks; First, it use signal strength as a measure of node mobility, which is not accurate with respect to noise obstacles, variation in power available and many other factors. Second, this scheme requires large communication overhead, also higher latency in cluster construction. So, it cannot evaluate stability of a node clearly. Summary of clustering algorithms listed in Table1. 
Table 1. Summary of Clustering Scheme for MANET

\begin{tabular}{|c|c|c|}
\hline Clustering Scheme & Characteristics & Cluster Head Election Criteria \\
\hline Lowest-ID [8] & Based on specified ID of the node & Node with lowest ID among 1-Hop \\
\hline DS Based Clustering [5][26] & $\begin{array}{c}\text { Cluster are formed based on } \\
\text { Dominating set of Nodes acting as } \\
\text { Cluster Heads }\end{array}$ & $\begin{array}{c}\text { Node must be member of Dominated Set to be } \\
\text { Cluster Head }\end{array}$ \\
\hline Highest Degree [8] & $\begin{array}{c}\text { Based on Highest Degree } \\
\text { (Connectivity Value) }\end{array}$ & $\begin{array}{c}\text { Highest connectivity value among its Direct } \\
\text { neighbors }\end{array}$ \\
\hline Mobility Based and Weight Clustering [2-3] & $\begin{array}{c}\text { Work similar to Lowest-ID with } \\
\text { tracking to node mobility }\end{array}$ & $\begin{array}{c}\text { Cluster head is the one with the lowest } \\
\text { aggregate mobility }\end{array}$ \\
\hline
\end{tabular}

\section{TPL Clustering Scheme}

The paper presents Threshold Power Level scheme (TPL), based on proactive routing protocol such as DSDV within the cluster [20] to keep track of nodes in cluster. Thus, it is not required to transmit explicit messages during cluster maintenance. The scheme use the local information, which means, less communication overhead and this directly translated into power efficiency. The proposed TPL scheme can be divided into two main phases; Cluster Formation Phase and Cluster Maintenance phase.

\subsection{Assumptions and Requirements}

Some nodes are elected to act as a Cluster Head in clustered network, and control nodes around them. Note that there may be a multiple Cluster Head for each cluster [22]; also, ordinary node can be a member for more than one cluster. Nevertheless, proposed scheme assumes that there is a single Cluster Head for each cluster. Also, any ordinary node is a member of single cluster at a time.

The proposed scheme modeled MANET as a set of nodes $\mathrm{SCN}=\{1 \ldots \mathrm{N}\}$ and Set of Cluster Head $\mathrm{SCH}=\{1 \ldots \mathrm{C}\}$, where $\mathrm{SCH} \subseteq \mathrm{SCN}$ and $\mathrm{N}$ is the total number of nodes in the cluster and $\mathrm{C}$ is the total number of candidate nodes that has opportunity to be elected as a Cluster Head. Previous work proposed that the set of nodes SCN remain static throughout the network lifetime but the cardinality of set of

Cluster Head i.e. $|\mathrm{CH}|_{\text {change due ESCN, where ESCN is }}$ energy considerations of the nodes in SCN. Also, the scheme assumes each node $\mathrm{n}_{\mathrm{i}} \in \mathrm{SCN}$ has:

- A Unique Integer Identifier $\mathrm{UII}_{\mathrm{i}}$.

- Cluster Head Flag $\mathrm{CHF}_{\mathrm{i}}$

- Current Energy $\mathrm{CE}_{\mathrm{i}}$.

- Threshold Total Energy Average TTEA

Where $\mathrm{CHF}_{\mathrm{i}}$ is a Boolean flag specifies whether the node was Cluster Head or not, $\mathrm{CE}_{\mathrm{i}}$ is the level of available energy in node $\mathrm{i}$, TTEA is the average of $\mathrm{CE}_{\mathrm{i}} \ldots \mathrm{n}$ for all nodes in the cluster.

\subsection{TPL Clustering Scheme}

The designated model gives a chance to all nodes in the cluster even those belong to $\mathrm{SCN}$ as ordinary node to be a Cluster Head and moved from SCN $\rightarrow$ SCH but it must specify election condition:

Any node can be Cluster Head iff

- $\quad \mathrm{CE}_{\mathrm{i}}>$ TTEA
The node to be elected not elected as Cluster Head in The current round

$$
\text { - } \mathrm{CHF}_{\mathrm{i}}==0
$$

Condition (1) indicates that all nodes must know the average energy of the entire network in TTEA parameter and use it as threshold to elect the Cluster Head. Also, every node must update the parameter calculation of TTEA and contain updated information about the power level in all nodes in that cluster. The idea behind this scheme is to balance the usage of power in every node belong to the cluster through using Round Robbins Scheduling techniques [24] which used to give every node same chance and a portion of time to be as a Cluster Head based on the level of energy it has $\mathrm{CE}_{\mathrm{i}}$ compared with the TTEA. The scheme works as follows:

Begin

\section{Step 1;}

For all $n_{i} \in S C N$ initialize $n_{i}$ parameters $U_{I I}$, $\mathrm{CHF}_{\mathrm{i}}, \mathrm{CE}_{\mathrm{i}}$

Insert $\mathrm{UII}_{\mathrm{i}}, \mathrm{CHF}_{\mathrm{i}}, \mathrm{CEi}$ into Cluster Table of $\mathrm{n}_{\mathrm{i}}$

Step 2;

For each node $\mathrm{n}_{\mathrm{i}} \in \mathrm{SCN}$ broadcast local parameters $\mathrm{UII}_{\mathrm{i}}, \mathrm{CHF}_{\mathrm{i}}, \mathrm{CE}_{\mathrm{i}}$

Step 3;

For each node $n_{j} \in S C N$ receives parameters for $n_{i}$ $\in \mathrm{SCN}$

* Insert into node $n_{j}$ Cluster Table the local parameter of ni at in place order (order based on $\left.C E_{i}\right)$

Step 4;

If $\mathrm{n}_{\mathrm{i}} \in \mathrm{SCN}$ is idle for time $\mathrm{T}$

Calculate TTEA $=\sum \frac{C E i}{N}$

Step 5;

For every node $n_{i} \in$ SCN start Cluster Head election process based on local Available information in all nodes

Begin

Find $\max \mathrm{CE}_{\mathrm{i}}$ in Cluster table and $\left(\mathrm{CHF}_{\mathrm{i}}=0\right)$

* if all CHFi are 1 rest all node flags. 
Assign the $\mathrm{UII}_{\mathrm{i}}$ of $\max \mathrm{CE}_{\mathrm{i}}$ as a Cluster Head

$$
\text { Set } \mathrm{CHF}_{\mathrm{i}}=1
$$

End

\author{
While (True) \\ If $\left(\mathrm{CE}_{\mathrm{i}}<\mathrm{TTEA}\right)$ Go to Step 1 ; End if
}

End while

End

The proposed scheme has the following feature: First, The Cluster Head always is the node with the maximum power which is theoretically maximize Mobile Ad Hoc Networks lifetime. Second, it gives all nodes the same chance to be a Cluster Head based on the power level available and the history of Cluster Head election. Third, Cluster Head is selected dynamically and minimize the amount of overhead needed in formation process while all data available in each node. This algorithm keeps track of the functionality of the MANET routing protocols.

\section{CONCLUSION}

This paper presents Cluster Head election algorithm to provide power efficiency technique to minimize the power usage in MANETs in theoretical view. The proposed technique is based on the average power in entire network compared with node power, which specify if it is a candidate for Cluster Head or not. The node with max power which has a chance to be a Cluster Head by status of uncounted as Cluster Head on entire network will be elected as a Cluster Head. It is clear that a node which has local power more than the average power of entire network may get a chance to be elected as Cluster Head. The proposed scheme saves battery energy by providing maximum number of the nodes a chance to act as Cluster Head and thereby saving any particular node to completely exhausted. Thus the future work aims to simulate the proposed scheme to check the value of performance can be added to MANETs by applying this clustering scheme.

\section{REFERENCES}

[1] Baker, D., Ephremides, A., \& Flynn, J. (1984). The Design and Simulation of a Mobile Radio Network with Distributed Control. IEEE Journal on Selected Areas in Communications, Vol. 2(No. 1), Pages 226-237.

[2] Basu, P., Khan, N., \& Little, T. D. C. (2001). A Mobility Based Metric for Clustering in Mobile Ad Hoc Networks. Presented at the Proceedings of the $21^{\text {st }}$ Inter. Conf. on Distributed Computing Systems, USA.

[3] Chen, G., Nocetti, F., Gonzalez, J., \& Stojmenovic, I. (2002). Connectivity Based k-hop Clustering in Wireless Networks. Paper presented at the Proceedings of the 35th Hawaii International Conference on System Sciences.

[4] Chen, G., \& Stojmenovic, I. (1999). Clustering and Routing in Wireless Ad Hoc Networks. Ottawa: Department of Computer Siences, SITE.

[5] Chen, Y. P., \& Liestman, A. L. (2002). Approximating minimum size weakly-connected dominating sets for clustering mobile ad hoc networks. Paper presented at the In Proceedings 3rd of the ACM International Symposium on Mobile Ad Hoc Networking and Computing (MOBIHOC'02), Switzerland.
[6] Chiang, C.-C., Wu, H.-K., Liu, W., \& Gerla, M. (1999). routing in clustered multi-hope, mobile wireless networks with fading channel. Paper presented at the (SICON'97) IEEE Singapore International Conference on Networks, Singapore.

[7] Dehni, L., Krief, F., \& Bennani, Y. (2005). Power Control and Clustering in Wireless Sensor Networks. Paper presented at the 4th Annual Mediterranean Ad Hoc Networking Workshop (IFIP), France.

[8] Ephremides, A., Wieselthier, J. E., \& Baker, D. J. (1987). A design concept for reliable mobile radio networks with frequency hopping signaling. Paper presented at the Proceeding of IEEE.

[9] Garcia, J.-E., Kallel, A., Kyamakya, K., Jobmann, K., Cano, J.-C., \& Manzoni, P. (2003). A novel DSR-based energy-efficient routing algorithm for mobile ad-hoc networks. Paper presented at the IEEE Vehicular Technology Conference, Florida, USA.

[10] Gerla, M., \& Tsai, J. (1995). Multicluster, mobile, multimedia radio network. ACM-Baltzer Journal of Wireless Networks, Vol.1 No.3, Pages 255-265.

[11] Ghanem, N., Boumerdassi, S., \& Renault, É. (2005). New energy saving mechanisms for mobile ad-hoc networks using OLSR. Paper presented at the PEWASUN '05 Proceedings of the 2nd ACM international workshop on Performance evaluation of wireless ad hoc, sensor, and ubiquitous networks, Canada.

[12] Johnson, D., \& Maltz, D. (1996). Dynamic source routing in ad hoc wireless networks. Paper presented at the In Imlellnski, T., Korth, H (eds.) Mobile Computing, Dordrecht.

[13] Kleinrock, L., \& Kamoun, F. (1977). Hierarchical routing for large networks: Performance Evaluation and Optimization. Computer Networks, Vol.1 No.1, 155-174.

[14] Krishnamurthy, S., \& Mohapatra, P. (2005). Ad Hoc networks Design Challenges and Future Opportunities. Business Media: Springer Science.

[15] Lee, S.-J., \& Gerla, M. (2001). Split multipath routing with maximally disjoint paths in ad hoc networks. Paper presented at the ICC2001 IEEE International Conference on Communications, Finland.

[16] Marina, M. K., \& Das, S. R. (2001). On-demand multipath distance vector routing in ad hoc networks. Paper presented at the ICNP 2001 9th International Conference on Network Protocols

[17] Meghanathan, N. (2007). Energy Consumption Analysis of the Stable Path and Minimum Hop Path Routing Strategies for Mobile Ad hoc Networks. IJCSNS International Journal of Computer Science and Network Security, Vol. 7(No. 10), Pages 30-39.

[18] Mishra, S., Satpathy, S., \& Mishra, A. (2010). Energy Efficient Design of Wireless Ad Hoc Networks. (Bachelor in Technology), National Institute of Technology, Rourkela. (769008)

[19] Perkins, C., \& Royer, E. (1999). Ad-Hoc on-Demand Distance Vector Routing. Paper presented at the Proceedings WMCSA'99. 2nd IEEE Workshop on Mobile Computing Systems and Apps, New Orleans. 
[20] Perkins, C. E., \& Bhagwat, P. (1994). Highly dynamic Destination-Sequenced Distance-Vector routing (DSDV) for mobile computers. Paper presented at the Proceeding SIGCOMM '94 Proceedings of the conference on Communications architectures, protocols and applications.

[21] Sarkar, S., Basavaraju, T. G., \& Puttamadappa, C. (2007). Ad Hoc Mobile Wireless Networks: Principles, Protocols and Applications. New York: Auerbach Publications.

[22] Tavli, B., \& Heinzelman, W. (2006). Mobile ad hoc networks: energy-efficient real-time data communications. Netherlands: Springer.

[23] Wei, D., \& Chan, H. A. (2006). Clustering Ad Hoc Networks: Schemes and Classifications. Sensor and $\mathrm{Ad}$
Hoc Communications and Networks. SECON '06. 3rd Annual IEEE Comm. Society, Vol. 03, PP 920-926.

[24] Wikipedia. [Online]. Wikipedia. [Online]. from http://en.wikipedia.org/wiki/Round_Robin_scheduling

[25] Wikipedia. [Online]. Wikipedia. [Online]. From http://en.wikipedia.org/wiki/Mobile_ad_hoc_network

[26] Wu, J., \& Li, H. (1999). On calculating connected dominating set for efficient routing in ad hoc wireless networks. Paper presented at the Proceeding DIALM '99 Proceedings of the 3rd international workshop on discrete algorithms and methods for mobile computing and communications, USA.

[27] Ye, Z., Krishnamurthy, S. V., \& Tripathi, S. K. (2003). A Framework for Reliable Routing in Mobile Ad Hoc Networks. USA. 